\title{
Influence of compensation status on time off work after carpal tunnel release and rotator cuff surgery: a meta-analysis
}

Vinícius Ynoe de Moraes ${ }^{1 *}$, Katelyn Godin², João Baptista Gomes dos Santos ${ }^{1}$, Flávio Faloppa?, Mohit Bhandari ${ }^{2}$ and João Carlos Belloti ${ }^{1}$

\begin{abstract}
Background: The assessment of post-surgical outcomes among patients with Workers' Compensation is challenging as their results are typically worse compared to those who do not receive this compensation. These patients' time to return to work is a relevant outcome measure as it illustrates the economic and social implications of this phenomenon. In this meta-analysis we aimed to assess the influence of this factor, comparing compensated and non-compensated patients.
\end{abstract}

Findings: Two authors independently searched MEDLINE (Ovid), Embase (Ovid), CINAHL, Google Scholar, LILACS and the Cochrane Library and also searched for references from the retrieved studies. We aimed to find prospective studies that compared carpal tunnel release and elective rotator cuff surgery outcomes for Workers' Compensation patients versus their non-compensated counterparts. We assessed the studies' quality using the Guyatt \& Busse Risk of Bias Tool. Data collection was performed to depict included studies characteristics and meta-analysis. Three studies were included in the review. Two of these studies assessed the outcomes following carpal tunnel release while the other focused on rotator cuff repair. The results demonstrated that time to return to work was longer for patients that were compensated and that there was a strong association between this outcome and compensation status - Standard Mean Difference, 1.35 (IC 95\%; 0.91-1.80, p<0.001).

Conclusions: This study demonstrated that compensated patients have a longer return to work time following carpal tunnel release and elective rotator cuff surgery, compared to patients who did not receive compensation. Surgeons and health providers should be mindful of this phenomenon when evaluating the prognosis of a surgery for a patient receiving compensation for their condition.

Type of study/level of evidence: Meta-analysis of prospective Studies/ Level III

Keywords: Workers' compensation, Hand surgery, Outcomes, Carpal tunnel syndrome, Rotator cuff tears, Systematic review, Time to return to work

\section{Introduction}

Numerous studies have demonstrated that Workers' Compensation patients have a longer return to work time compared to non-compensated patients [1-3]. Other studies have described the inherent difficulties

\footnotetext{
* Correspondence: vymoraes@gmail.com

'Division of Hand and Upper Limb Surgery, Department of Orthopaedics and Trauma, Universidade Federal de São Paulo (UNIFESP-EPM), Rua Borges Lagoa, 778, São Paulo, Brazil

Full list of author information is available at the end of the article
}

associated with measuring time to return to work as an outcome of the success of a surgical treatment [4-6].

These findings are of relevance when a surgeon is attempting to predict a patient's loss of working days and the accompanying economical and social implication [7]. There are numerous factors that have been shown to determine return to work time including psychological issues, job type and work-place features $[1,4,5,8,9]$.

We believe that is important to understand and quantify the difference in return to work time between

\section{Biomed Central}




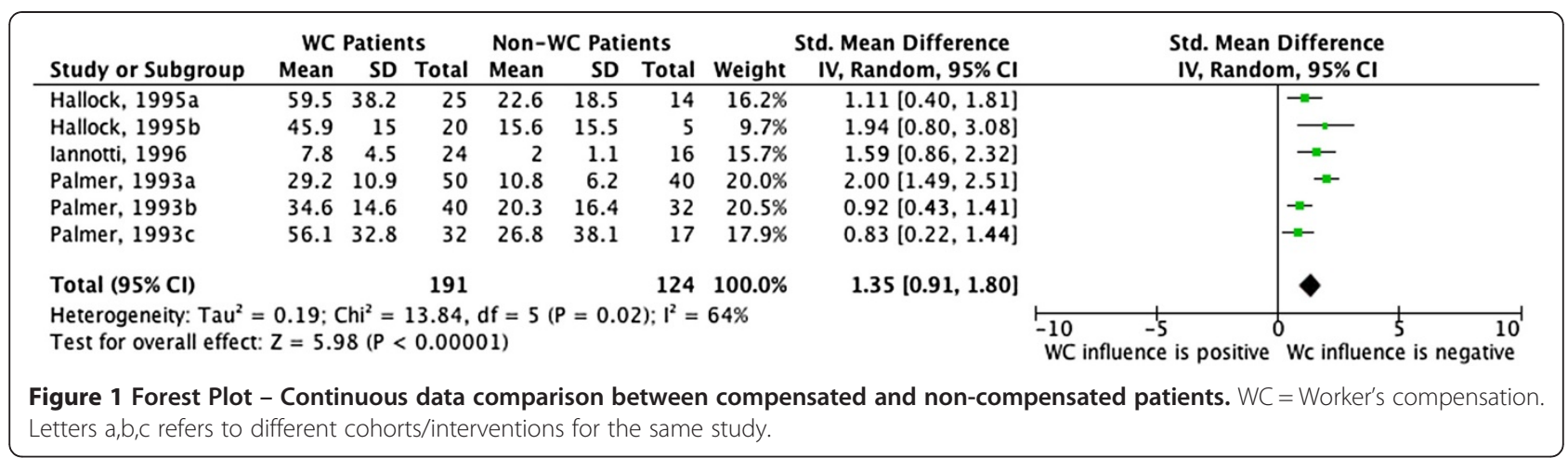

compensated and non-compensated patients. We did not recognize this approach based in a Systematic Review and Meta-Analysis including only a best-evidence approach.

This systematic review and meta-analysis aims to analyze time to return to work as reported from data gathered from prospective studies that assessed patient outcomes following carpal tunnel release and elective rotator cuff surgery.

\section{Methods}

This study is a complementary analysis from a broader systematic review that assessed the results of the compensation status following orthopedic surgery [10] and considered functional outcomes as the main endpoint. The endpoints assessed in this report were set a priori and the protocol was published before study began in a prospective database for systematic reviews (http://www. crd.york.ac.uk/prospero) under the record number CRD42012002121 [11].

\section{Search strategy and assessment of eligible studies}

The following databases were searched: MEDLINE (Ovid), Embase (Ovid), CINAHL, Google Scholar, LILACS and the Cochrane Library. We also hand-searched the references sections of these primary papers in order to locate additional studies and to avoid missing relevant papers. We did not exclude any studies on the basis of language.

We included papers published between 1992 and 2012 (May, 2012). Our search strategy is shown in Figure 1.

\section{Selection criteria}

Studies were included if they met the following criteria: 1) the data was collected and analyzed prospectively; 2) the authors assessed the influence of compensation status specifically; 3) orthopaedic surgery was the main intervention. In this report, we included only the studies that reported time to return to work after upper limb orthopaedic surgery (carpal tunnel release and elective rotator cuff surgery). We excluded studies that: 1) involved non-surgical treatments; 2) studies that collected patient data retrospectively; and 3) the study did not report any of the outcomes of interest, as described above. We included studies after a 2-stage assessment. Disagreements regarding which studies should be included were resolved by group discussion (VY and KG).

\section{Data management: collection and extraction}

Quantitative data was extracted following full text analysis of the included studies. Other important information that was extracted included details on the study design, funding, intervention, control (if applicable) and outcomes.

We collected data (VY, KG) as continuous and analyses were performed in forest plots. Time to return to work data was collected from reported means, standard deviations, and the number of patients in each group. In the event that the published data was missing and/ or unclear, we also attempted to contact the authors by email to clarify or provide us with additional data from their study. Following data entry, all data was verified by two authors (VY and JB).

Table 1 Studies characteristics: qualitative data

\begin{tabular}{lllll}
\hline Study & Intervention & Country & Study design & Funding \\
\hline Palmer [15] 1993 & Carpal Tunnel Release & US & Prospective Case Series & No \\
\hline Hallock [2] 1995 & Carpal Tunnel Release & US & Prospective Case Series & No \\
\hline lannotti [3] 1996 & Rotator Cuff Tear Repair & US & Prospective Case Series & No \\
\hline
\end{tabular}


Table 2 Studies characteristics: quantitative data

\begin{tabular}{llllll}
\hline Study & Participants (n) & Intervention & Gender (\%) & Age (SD) & Follow up losses (\%) \\
\hline Palmer [15]1993 & 163 & Carpal Tunnel Release & 73(44.7\%) & $42.2-46.8$ & 0 \\
\hline Hallock [2] 1995 & 96 & Carpal Tunnel Release & 26(27\%) & $45.6(17) / 42.6(14)$ & 0 \\
\hline lannotti [3] 1996 & 46 & Rotator Cuff Tear Repair & $31(77.5 \%)$ & $55(11)$ & 13 \\
\hline
\end{tabular}

\section{Study quality assessment}

We assessed the quality of all included studies using a specific tool [12] developed to appraise the risk of bias within observational studies. Each included study was graded on a four-category scale according to their risk of bias. The scale and its grading scheme is shown in Additional file 1: Appendix 1.

\section{Statistical analysis}

We utilized RevMan [13] (version 5.1) to conduct the meta-analysis. The data was summarized using an Inverse Variance method in a Random Effect model. We provided measures as standardized mean difference [14], as proposed by Hedges. We followed Cohen's interpretation on the magnitude of effect size: small effect $<0.20$, medium effect $<0.50$ and large effect $<0.80$. We provided $95 \%$ confidence intervals shown in forest plots for each of the included studies and also for the sum of the studies [14]. Heterogeneity was assessed by $\mathrm{I}^{2}$ statistics and is depicted in a forest plot.

\section{Results}

Using our search strategy, we analyzed the title and abstract of 791 studies for relevance to our study. We assessed the full text of 67 studies and 20 met our inclusion criteria. We identified three studies that assessed time to return to work in patients who were compensated versus those who did not receive compensation. The analyses included 315 patients. The studies' characteristics are presented in Tables 1 and 2. Two studies were excluded because they did not report the data we required. Despite our efforts to retrieve this information from authors, we could not obtain this data. The quality assessment demonstrated that methodological quality differed among studies. One study was evaluated as having a low risk of bias [3], one has as intermediate risk [15] and one as high risk [2]. Figure 2 demonstrates a strong effect size for the relationship between time to return to work and compensation status, with compensated patients having a longer return to work time.

Search Strategy: EMBASE (OVID) \& MEDLINE (OIVD) -

1 workers compensation.mp. or exp Workers' Compensation/

2 work\$ compensation.mp. (7523)

31 or 2

4 exp Arthroplasty, Replacement/ or exp Orthopedic Procedures/or exp Orthopedics/or exp Pain, Postoperative/ or orthopaedic surgery.mp. or Fractures, Bone

$5 \quad 3$ and 4

$* * * * * * * * * * * * * * * * * * * * * * * * * * *$

Limits=from 1992 to 2012 .

For the remaining databases: CINAHL, LILACS, Cochrane Library, Google Scholar

Free search for the words:

1. workers compensation AND

2. orthopedic surgery

$* * * * * * * * * * * * * * * * * * * * * * * * * * *$

Limits=from 1992 to 2012.

Figure 2 Search strategy. 


\section{Discussion}

Our results demonstrate that compensated patients had a longer time of absence from work following orthopedic surgery. Although this finding may be intuitive, this study demonstrates this relationship using a rigorous, systematic perspective.

Some studies have previously demonstrated that time to return to work is not an optimal measure for surgeons when assessing post-surgery outcomes $[4,16,17]$. However, return to work time is an important consideration as it contributes to social and economical burden [6].

Factors that influence earlier or later return to work are well-established in the literature and typically relate to the particular health condition, surgical intervention, the surgeon's approach for post-operative treatment and care and patient characteristics $[1,4]$. This study demonstrates that the presence of Worker's Compensation is an additional factor that predicts a longer return to work period post-surgery.

This meta-analysis provides quantitative data as Standardized Mean Difference. This is not a directly usable measure, as it measures the standardized difference between the standard deviations between the groups of interest. This measure is frequently used in Cochrane Reviews when different scales are pooled in the same forest plot. In our study, this quantification allows us to state that compensated patients take longer to return to work compared to non-compensated patients and the magnitude of this effect is strong [14].

The small number of included studies and the lack of studies including different conditions reduced the external validity of our results as our findings may not be generalizable to other patient populations. We opted not to include lower limb diseases or acute injuries (e.g. fractures) since this would increase the heterogeneity of our results in an inacceptable manner for our outcome of interest. This study is strengthened by the robustness of the methodology, the strict inclusion criteria and quality assessment.

\section{Conclusions}

Compensated patients are more likely to have longer periods of recovery after carpal tunnel release and elective rotator cuff surgery. This finding has significant clinical and economical implications. Prospective studies are the best way to determine which factors influence return to work time following surgery.

\section{Additional file}

Additional file 1: Appendix 1. Tool to Assess Risk of Bias in Cohort Studies.

\section{Competing interest}

No additional external funding received for this study. The authors declare that they have no competing interests. The funders had no role in study design, data collection and analysis, decision to publish, or preparation of the manuscript.

\section{Authors' contributions}

VYM and KG wrote and JCB reviewed the manuscript. WYM developed the idea for the study. WYM and KG carried out the study's assessment. VYM performed the statistical analysis and MB provided research support. JBGS and FF provided additional research support and were involved in the manuscript proofreading. WYM and KG revised the manuscript. All authors have read and approved the final manuscript.

\section{Acknowledgement}

Dr. Bhandari is funded, in part, by a Canada Research Chair. Part of the funding for this study was provided by FAPESP Fundação de Amparo a Pesquisa do Estado de São Paulo, Brazil, Process number: 11/21611-2.

\section{Author details}

${ }^{1}$ Division of Hand and Upper Limb Surgery, Department of Orthopaedics and Trauma, Universidade Federal de São Paulo (UNIFESP-EPM), Rua Borges Lagoa, 778, São Paulo, Brazil. ²Division of Orthopaedic Surgery, Department of Surgery, McMaster University, 293 Wellignton St. N, Hamilton, ON L8L 8E7, Canada.

Received: 30 November 2012 Accepted: 27 December 2012 Published: 2 January 2013

\section{References}

1. Adams ML, Franklin GM, Barnhart S: Outcome of carpal-tunnel surgery in Washington-state workers compensation. Am J Ind Med 1994, 25:527-536.

2. Hallock GG, Lutz DA: Prospective comparison of minimal incision "open" and two-portal endoscopic carpal tunnel release. Plast Reconstr Surg 1995, 96:941-947.

3. lannotti JP, Bernot MP, Kuhlman JR, Kelley MJ, Williams GR: Postoperative assessment of shoulder function: a prospective study of full-thickness rotator cuff tears. J Shoulder Elbow Surg 1996, 5:449-457.

4. Cowan J, Makanji H, Mudgal C, Jupiter J, Ring D: Determinants of return to work after carpal tunnel release. J Hand Surg-Am 2012, 37A:18-27.

5. Parot-Schinkel E, Roquelaure Y, Ha C, Leclerc A, Chastang JF, Raimbeau G, Chaise F, Descatha A: Factors affecting return to work after carpal tunnel syndrome surgery in a large french cohort. Arch Phys Med Rehab 2011, 92:1863-1869.

6. MacDermid JC, Grewal R, MacIntyre NJ: Using an evidence-based approach to measure outcomes in clinical practice. Hand Clin 2009, 25:97-101.

7. Mallick A, Clarke M, Wilson S, Newey ML: Reducing the economic impact of carpal tunnel surgery. J Hand Surg-Eur Vol 2009, 34E:679-681.

8. Acharya AD, Auchincloss JM: Return to functional hand use and work following open carpal tunnel surgery. J Hand Surg-Brit Eur 2005, 30B:607-610.

9. Bruyns CNP, Jaquet JB, Schreuders TAR, Kalmijn S, Kuypers PDL, Hovius SER: Predictors for return to work in patients with median and ulnar nerve injuries. J Hand Surg-Am 2003, 28A:28-34.

10. de Moraes VY, Godin K, Tamaoki MJS, Faloppa F, Bhandari M, Belloti JC: Workers' Compensation status: does It affect orthopaedic surgery outcomes? A meta-analysis. PLoS One 2012, 7:e50251.

11. Moraes VY, Belloti JC, Godin K, Bhandari M: Influence from presumed compensation status in orthopedic surgery: does it affect the outcomes? a systematic review of prospective studies. PROSPERO Database 2012, CRD42012002121.

12. Guyatt G, Busse J: Tool to assess risk of bias in cohort studies. Hamilton, ON: In Book Tool to Assess Risk of Bias in Cohort Studies; 2011.

13. RevMan R: The nordic cochrane centre, the cochrane collaboration, Book [computer program]. version 5.1. Copenhagen: The nordic cochrane centre. Version 51st edition. Copenhagen: The Cochrane Collaboration; 2011.

14. Durlak JA: How to select, calculate, and interpret effect sizes. J Ped Psych 2009, 34:917-928. 
15. Palmer DH, Paulson JC, Lanelarsen CL, Peulen VK, Olson JD: Endoscopic carpal-tunnel release - a comparison of 2 techniques with open release. Arthroscopy 1993, 9:498-508.

16. Atroshi I, Johnsson R, Ornstein E: Patient satisfaction and return to work after endoscopic carpal tunnel surgery. J Hand Surg-Am 1998, 23A:58-65.

17. Baldwin ML, Johnson WG, Butler RJ: The error of using returns-to-work to measure the outcomes of health care. Am J Ind Med 1996, 29:632-641.

doi:10.1186/1754-9493-7-1

Cite this article as: de Moraes et al.: Influence of compensation status on time off work after carpal tunnel release and rotator cuff surgery: a meta-analysis. Patient Safety in Surgery 2013 7:1.

\section{Submit your next manuscript to BioMed Central and take full advantage of:}

- Convenient online submission

- Thorough peer review

- No space constraints or color figure charges

- Immediate publication on acceptance

- Inclusion in PubMed, CAS, Scopus and Google Scholar

- Research which is freely available for redistribution 